\title{
RÉSISTANCE DES MATÉRIAUX
}

\section{Le calcul des tubes à section elliptique soumis à une pression intérieure}

par Frank F. Fergussox, A. M. I. C. E., F. G. S., Ingénieur aux Etablissements J. Blakeborough \& Sons England.

Deux méthodes ont été exposées dans la Houille Blanche $N^{\prime 0} 172$ page 16\%, par M. l'ingénieur G. Prudon et dans le $\Upsilon^{\circ}$ 1\% 3 , page 213, par M. l'ingénieur P. Cayère, pour le calcul des fatigues du métal composant l'enveloppe alliptique des corps de robinets vannes.

L'application de ces méthodes, en détail, exige une dépense de temps assez grande et le soussigné croit qu'il sera peut-itre utile aux ingénieurs-dessinateurs de donner ses expériences gagnées dans le calcul d'un fort grand nombre de sections élliptiques.

L'examen des cas nombreux par les méthodes exposées par MN. Prudon et Cayère a démontré que le moment fléchissant positif maximum, $\mathrm{M} b$, au bout de l'axe mimor est, dans chaque cas, égal à

$$
\frac{\mathrm{P}}{2} \times b^{\mathrm{a}} \times J_{\mathrm{b}} \mathrm{ks} . \mathrm{cm}
$$

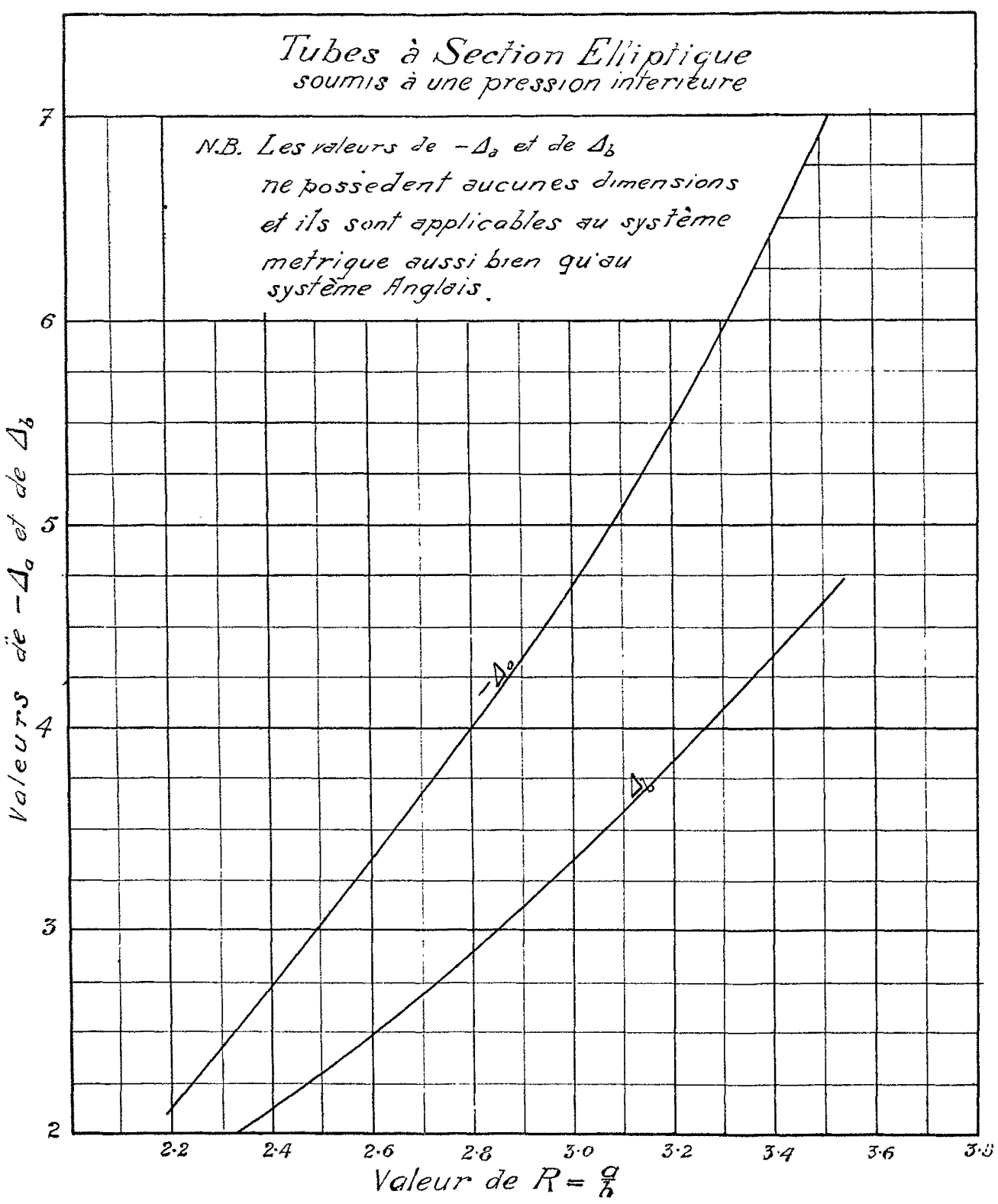

Fig. 1
$\mathrm{P}=p d \mathrm{~kg} . \mathrm{cm}$.

$\mathrm{p}=$ la pression intéricure en $\mathrm{kg} / \mathrm{cm}^{2}$

$d=$ la distance entre les centres des nervures horizontales.

$$
s_{1}=\left(a^{2}-b^{2}\right)-\frac{\Xi\left(a^{2}-r^{2}\right)}{n}
$$

$a=$ la longueur de l'axe semi-major.

$b=$ la longueur de l'axe semi-minor.

Dans l'équation (2) $a$ et $b$ ont la valeur de $\mathrm{R}$ el de l'unité, où $\mathrm{R}$ est le rapport entre les deux axes, $2 a$ et $2 b$, et $n$ est le nombre des valeurs de $r$ dans lequel on divise la circonférence del'enveloppe $\rfloor_{b}$ est clairement une valeur constante pour une valeur donnée de $R$.

Semblablement, le moment fléchissant négatif maximum, $-\mathrm{M}_{\mathrm{a}} \mathrm{kg} . \mathrm{cm}$, au bout de l'axe major est égal à :

$$
\frac{\mathrm{P}}{\mathrm{g}} \times b^{2} \times\left(-\Delta_{\mathrm{a}}\right)
$$

ou

$$
-\lrcorner_{\mathrm{a}} \frac{\sum\left(a^{2}-\gamma^{2}\right)}{n}
$$

$a$ et $b$ étant $\mathrm{R}$ et unité comme susdil, afin qu'on puisse éviter de multiplier par $b^{2}$ tous les termes du calcul.

On peut voir maintenant qu'on n'a qu'à prendre les valeurs de $M_{b}$ et de $-M_{a}$ et de les diviser par $\frac{\mathrm{P}^{2}}{2}$, et avec ces résultats on peut construire une représentation graphique. En posant les valeurs de $-\Delta_{\mathrm{a}}$ et de $\Delta_{\mathrm{b}}$ contre l'axe de $y$ et celles de $\mathrm{R}$ contre l'axe de $x$, voir fig. 1.

Par moyenne de ces courbes on peut déterminer les valeurs des moments fléchissants maximum positifs et négatifs pour aucun cas, quoi que ce soil, par une multiplication sur la règle de calcul, qui permettra de préciser une section convenable pour résister aux efforts. 
Exentple : Pression intérieure $=5,12$ atmosphères;

Longucur de l'axe major $=2780 \mathrm{~m} / \mathrm{m}$;

Longueur de l'axe minor $=1112 \mathrm{~m} / \mathrm{m}$.

$$
\begin{gathered}
\mathrm{R}:=\frac{\frac{9}{2} a}{2 b}=\frac{2780}{1112}=2,5 \\
a=\frac{2780}{2}=139() \mathrm{m} / \mathrm{m} \quad b=5.56 \mathrm{~m} / \mathrm{m} \quad b^{2}=3091365 \\
p=5,12 \mathrm{~kg} . \mathrm{cm} .
\end{gathered}
$$

Admettant pour $d$ une valeur de $300 \mathrm{~m} / \mathrm{m}$, on aura :

$$
\begin{gathered}
P=5,12 \times 30=153,6 \mathrm{~kg} . \mathrm{cm} . \\
\frac{P}{2}=76,8 \mathrm{~kg} . \mathrm{cm} .
\end{gathered}
$$

D'après les courbes de fig. 1 , on voit que

$$
\begin{aligned}
\Delta_{b} & =2,3 ; \\
-\Delta_{a} & =-3,0 ;
\end{aligned} \text { pour une valeur de } r=2,5
$$

$\cdot M_{\mathrm{b}}=J_{\mathrm{b}} \cdot \frac{\mathrm{P}}{2} \cdot b^{2}=-2,3 \times 7+\mathrm{i}, 8 \times 30991,36=54 \mathrm{t}, 058 \mathrm{~kg} \cdot \mathrm{cm}$. et

$-\mathrm{M}_{\mathrm{a}}=-\Delta_{\mathrm{a}} \cdot \frac{\mathrm{P}}{2} \cdot b^{2}=3,0 \times 76,8 \times 3(091,36=712,249 \mathrm{~kg} \cdot \mathrm{cm}$.

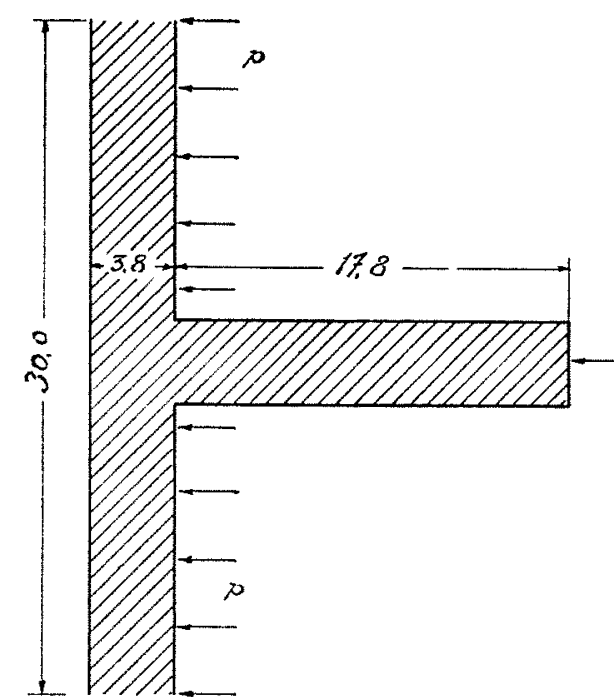

COUPE TRANSUERSALE aU BOUT de IAXE MINOR.

L'effort d'extension au bout de l'axe " $a$ » est égal à :

$$
\mathrm{T}_{\mathrm{a}}=\mathrm{P} a=153,6 \times 139=21,350 \mathrm{~kg} \text {. }
$$

et au bout de l'axe $b$ :

$$
\mathrm{T}_{\mathrm{b}}=\mathrm{P} b=15.3,6 \times 55,6=8540 \mathrm{~kg}
$$

il ne reste maintenant qu'à choisir une section qui convient le mieux pour résister économiquement aux efforts et en même lemps qui conserve une forme pratique au point de vue de labrication.

Le soussigné a toujours usé d'une section de la forme de fig. 5 de l'exposé de $M$. Cayère avec de bons résultats.

Considérons une tranche de l'enveloppe de $30 \mathrm{cms}$ de longueur avec une nervure dans le centre; voir fig. 2 , et prenant d'abord la coupe transversale au bout de l'axe minor, on aura :
L'aire

$$
=30 \times 3,81+17,8 \times 3,81=182,12 \mathrm{~cm}^{2}=A_{\mathrm{b}}
$$

Moment d'inertie

$$
=6768 \mathrm{~cm}^{2}=\mathrm{I}_{\mathrm{b}}
$$

Moment de résistance

. Effort

$$
=\frac{6768}{5,92}=1143=Z_{\mathrm{b}}
$$

$$
=\frac{M_{\mathrm{b}}}{Z_{\mathrm{b}}}=\frac{546,058}{114.9}=477,7 \mathrm{~kg} \cdot \mathrm{cm}^{2}
$$

Effort direct

$$
=\frac{\Gamma_{\mathrm{b}}}{\mathrm{A}_{\mathrm{b}}}=\frac{8540}{187,17}=47 \mathrm{~kg} \cdot \mathrm{cm}^{2}
$$

. Effort total d'extension

$$
=477,7+47=0.24,7 \mathrm{~kg} \cdot \mathrm{cm}^{2}
$$

Les efforts au bout de l'axe major :

L'aire

$$
=30 \times 3,81+22,86 \times 3,81=201,30 \mathrm{~cm}^{2}=A_{a}
$$

Moment d'inertie

$$
=12,759=\mathrm{I}_{\mathrm{a}}
$$
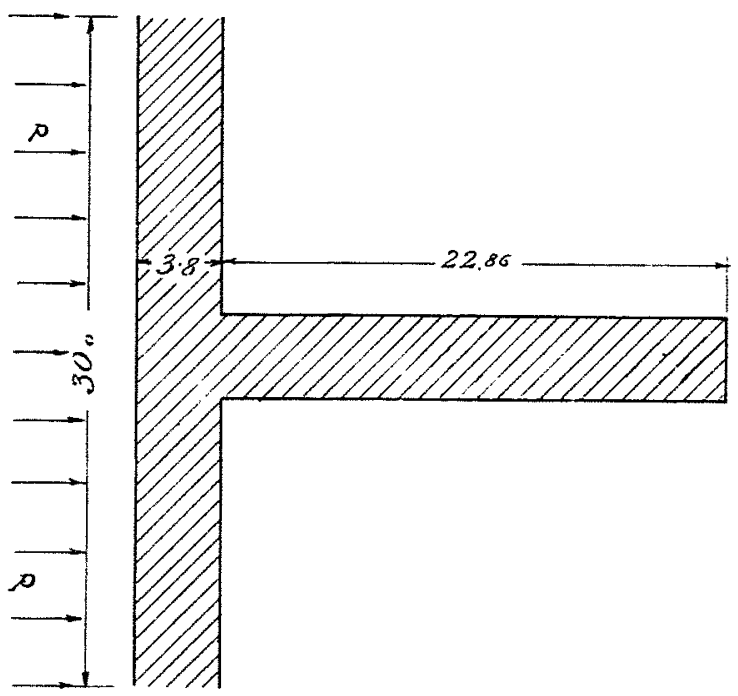

\section{COUPE TRANSUERSALE \\ CU BOUT del'AXE MAJOR.}

Moment de résistance

. Effort

$$
=\frac{12,59}{2(1,59}=16166=Z_{\mathrm{a}}
$$

$$
=\frac{712,2 \cdot 49}{1666}=427 \mathrm{~kg} \cdot \mathrm{cm}^{2}
$$

Effort direct

$$
=\frac{21,350}{201,39}=106 \mathrm{~kg} \cdot \mathrm{cm}^{2}
$$

- Effort total d'extension

$$
=427+106=533 \mathrm{~kg} \cdot \mathrm{cm}^{2}
$$

Les calculs donnés dans cet exemple sont pour le cas actuel d'une vanne pour conduite forcée de $2400 \frac{\mathrm{m}}{\mathrm{m}}$ de diamètre. 